\title{
Helium-film-induced retrapping transition in the two-dimensional electron system above an uneven solid-hydrogen surface
}

\author{
Yuri P. Monarkha \\ Institute for Low Temperature Physics and Engineering, 47 Lenin Avenue, Kharkov, 310164 Ukraine \\ Uwe Albrecht, Kimitoshi Kono, ${ }^{*}$ and Paul Leiderer ${ }^{\dagger}$ \\ Fakultät für Physik, Universität Konstanz, D-7750 Konstanz, Federal Republic of Germany
}

(Received 31 August 1992)

\begin{abstract}
A detailed theoretical analysis of the conductivity of surface electrons above a thin liquid-helium film covering an uneven solid-hydrogen surface is presented. The pronounced conductivity anomaly which is observed during the growth of the helium film is shown to originate from a strong coupling of the electrons to the roughness of the underlying hydrogen substrate. The observed conductivity anomalies (dip and peak) can be explained quantitatively by a retrapping structural transition within the disordered system of localized charges. We have measured the effects of varying electron density and discuss the influence of electronic correlation on the conductivity.
\end{abstract}

\section{INTRODUCTION}

One of the most intriguing results in studies of surface electrons (SE's) above helium films is the observation of a pronounced anomaly in the conductivity upon a continuous change in film thickness. This anomaly was first reported by Andrei ${ }^{1}$ for rather thick saturated ${ }^{4} \mathrm{He}$ films $(d \simeq 900 \AA)$ covering a sapphire substrate. A similar phenomenon has been observed on solid neon ${ }^{2}$ and solid-hydrogen substrates, ${ }^{3-5}$ and it has been extensively studied on quench-condensed hydrogen films. ${ }^{6}$ The results obtained on different substrates look strikingly similar, although it is not clear whether they represent the same effect.

Several explanations for this conductivity anomaly have been proposed. It has first been ascribed to electron-ripplon scattering. ${ }^{3}$ However, at temperatures around $2 \mathrm{~K}$ where it typically is observed this contribution is rather weak, and it is not clear why it should lead to such a pronounced structure. A resonance enhancement due to the ac-conductivity measurements can also be excluded. ${ }^{6}$ A possible polaronic state which has been discussed as an origin of the anomaly ${ }^{6,7}$ would have a binding energy much smaller than $k_{B} T$ under the experimental conditions.

An alternative explanation has been presented recently. $^{8}$ There the interaction of the electrons with the roughness of the underlying substrate is considered as a function of helium-film thickness. It is found to change sign at a definite thickness value $d=d^{*}$ and attains a local extremum at $d>d^{*}$. In this paper we present the results of SE conductivity studies in the limit of strong coupling of the electrons with surface irregularities, and compare them to experimental data.

In Sec. II the theoretical concept is outlined and the relevant equations for the electron-roughness interaction are derived. Section III then deals with the consequences of our results for experimental systems: in Sec. III A we explicitly calculate the interaction potential and evaluate the electron conductivity for appropriate experimental parameters; the results are compared with experimental data in Sec. III B; in Secs. III C and III D we examine the influence of changes in the roughness wave vector and of correlation effects within the electron system. Section IV summarizes the conclusions drawn from our study.

\section{THEORETICAL CONCEPT}

The SE conductivity anomaly we are attempting to explain can be observed at temperatures as high as $2 \mathrm{~K}$. Thus we can neglect the electron-ripplon scattering and take into account only the static equilibrium distortion of the helium surface caused by an uneven solid substrate. Experiments ${ }^{6}$ which exhibit the anomaly have shown the SE conductivity on bare hydrogen to be of thermally activated nature with rather large activation energies $\left(\Delta E_{s} \simeq 10 \mathrm{~K}\right)$. We therefore consider the coupling between the $\mathrm{SE}$ and the substrate roughness to be strong, i.e., the electrons are mostly trapped at surface irregularities with their mobility being dominated by thermally activated hopping.

A helium film changes the interaction of the SE with the roughness of the solid substrate in two qualitative aspects. It lifts the electrons away from the surface irregularities, reducing the interaction with the substrate, and it smooths the surface on which they move. It seems that both these effects should monotonously reduce the interaction with the roughness. However, a consistent treatment shows that a reduction only takes place within certain ranges of the film thickness.

In order to find the Fourier transform $V_{q}^{(f)}$ of the interaction potential of the SE with the uneven solid substrate in the presence of a helium film, we must consider that the electronic motion is confined to the film 
surface at $z=\xi^{(l)}(\mathbf{r})$. In this case the electron wave function distorted by the uneven helium-film surface differs substantially from the one above a flat surface $\langle 1 \mid z\rangle$. On the uneven surface it is actually close to $\left\langle 1 \mid z-\xi^{(l)}\right\rangle$ and it is generally accepted to use the matrix elements of the interaction potential with the unperturbed wave functions in this case, changing the perpendicular coordinate from $z$ to $z+\xi^{(l)}$. Accounting for the polarization attraction between the electrons and the helium-covered solid substrate in the usual way ${ }^{8}$ and inserting the abovementioned transformation of the $z$ coordinate, the linear terms of $V_{q}^{(f)}$ can be written as follows:

$$
\begin{aligned}
V_{q}^{(f)}= & {\left[e E_{\perp}+P_{q}^{(l)}(z)\right] \xi_{q}^{(l)} } \\
& +\frac{\Lambda_{s}-\Lambda_{l}}{(z+d)^{2}}\left\{\xi_{q}^{(l)} / \xi_{q}^{(s)}-q(z+d) K_{1}[q(z+d)]\right\} \xi_{q}^{(s)}
\end{aligned}
$$

Here $\xi_{q}^{(p)}$ denotes the Fourier transform of the liquidhelium $(p=l)$ or solid-hydrogen $(p=s)$ surface profile [the index $(f)$ stands for quantities relating to the helium film with thickness $d$ on the substrate], $e$ is the electron charge, $E_{\perp}$ is the electric holding field, $q$ is the surfaceroughness wave number, $K_{n}(x)$ is the modified Bessel function, and

$$
\Lambda_{p}=\frac{e^{2}\left(\epsilon_{p}-1\right)}{4\left(\epsilon_{p}+1\right)}, \quad p=s, l .
$$

Equation (1) exhibits the necessary limiting behavior for the helium-film thickness $d \rightarrow \infty$ and for $d \rightarrow 0$. The first term $V_{q}^{(l)}=\left[e E_{\perp}+P_{q}^{(l)}(z)\right] \xi_{q}^{(l)}$ in Eq. (1) is well known from the electron-ripplon interaction potential and describes the interaction of the electron with static deformations of the helium surface. Its polarization part $P_{q}^{(l)}(z)$ has the specific form

$P_{q}^{(l)}(z)=\frac{1}{2} \Lambda_{l} q^{2} Q(q z), \quad Q(x)=\frac{2}{x^{2}}\left[1-x K_{1}(x)\right]$.

This term decreases substantially in the long-wavelength limit $q z \ll 1$, with $Q(x) \simeq \ln (2 / \gamma x)+1 / 2 \ll 2 / x^{2}$ (here $\gamma \simeq 1.78$ ), since $K_{1} \rightarrow 1 / x$ for $x \ll 1$. Below we will show that this important behavior leads to a strong influence of even a thin helium film covering the uneven solid substrate.

For $d \rightarrow 0$ the interaction potential $V_{q}^{(f)}$ obviously transforms continuously into the potential for the interaction with the bare hydrogen surface irregularities,

$$
V_{q}^{(f)}(z, 0)=V_{q}^{(s)}(z) \equiv\left[e E_{\perp}+P_{q}^{(s)}(z)\right] \xi_{q}^{(s)},
$$

since the ratio of the Fourier-transformed surface profiles, which can be written as ${ }^{8}$

$B_{q} \equiv \xi_{q}^{(l)} / \xi_{q}^{(s)}=\frac{0.5(q d)^{2} K_{2}(q d)}{1+\left(d / d_{0}\right)^{4}}, \quad d_{0}^{4}=\frac{3 A_{H} \rho_{l}}{\alpha_{l} m_{\mathrm{He}} q^{2}}$,

rapidly becomes equal to unity for $d \ll d_{0}$ and $q d \ll 1$ (here $A_{H}$ is the van der Waals-Hamaker constant; $\rho_{l}$, $\alpha_{l}$, and $m_{\mathrm{He}}$ are the density, surface tension, and atomic mass of helium, respectively).

The polarization term $P_{q}^{(l)}(z)$ is always positive for a bulk liquid. This means that in the strong-coupling limit the electrons are trapped in the "valleys" of the surface profile $\xi^{(l)}(\mathbf{r})$. The same holds for the bare hydrogen surface, since $P_{q}^{(l)}$ differs from $P_{q}^{(s)}$ only by a constant factor $\Lambda_{l} / \Lambda_{s}$. Now considering a helium film on the uneven hydrogen substrate it can easily be seen that at $d \ll d_{0}$ the surface smoothing effect on the electronic motion is negligible $\left(\xi^{(l)} \simeq \xi^{(s)}\right)$ and the second polarization term in Eq. (1) is proportional to $Q[q(z+d)]>0$. Consequently for such a thin film the electrons are still trapped in the valleys of the surface profile. However, the smoothing effect becomes of major importance in the intermediate regime where $d \simeq d_{0}$. The ratio of the profiles $B_{q}=\xi_{q}^{(l)} / \xi_{q}^{(s)}$ starts to be substantially smaller than 1 , since $B_{q}=1 /\left[1+\left(d / d_{0}\right)^{4}\right]$ (for $q d \ll 1$ ) is rapidly decreasing with $d$. According to Eq. (4) $d_{0}$ is proportional to $q^{-1 / 2}$. Such a behavior destroys the balance of the polarization terms in Eq. (1). Hence the large negative asymptote $-x K_{1}(x) \simeq-1$ is not compensated any more, thus at some point the interaction potential must change sign. This can be seen more easily by rewriting Eq. (1) as

$$
V_{q}^{(f)}(z, d)=\left[e E_{\perp} B_{q}+P_{q}^{(f)}(z, d)\right] \xi_{q}^{(s)},
$$

where

$$
\begin{aligned}
P_{q}^{(f)}(z, d)=\frac{1}{2} \Lambda_{s} q^{2}[ & (1-\lambda) B_{q} Q(q z) \\
& \left.+\lambda Q[q(z+d)]+\frac{2 \lambda\left(B_{q}-1\right)}{[q(z+d)]^{2}}\right],
\end{aligned}
$$

with $\lambda=1-\Lambda_{l} / \Lambda_{s} \simeq 0.783$. In the long-wavelength limit we have $Q[q(z+d)] \ll 2 /[q(z+d)]^{2}$, so that already a small disbalance $B_{q}<1$ in the last term of Eq. (5) can lead to a negative $P_{q}^{(f)}$, eventually changing the sign of $V_{q}^{(f)}$. The absolute value of the potential $V_{q}^{(f)}$ may then even increase beyond the value for the bare hydrogen film upon increasing $d$ further.

The physical picture behind the change in sign of the interaction potential is schematically sketched in Fig. 1. It marks a transition from the localization of the SE in the valleys of the surface irregularities for thin helium films [Fig. 1(a)] to the situation for a thick, nearly flat helium film, where the electrons are localized opposite the tops of the solid surface profile $\xi^{(s)}(\mathbf{r})$ [Fig. 1(b)]. In between these two states where the SE's are trapped, there must be a transition at some film thickness $d=$ $d^{*} \simeq d_{0}$ where they are virtually free [i.e., $V_{q}^{(f)}\left(z, d^{*}\right)=$ $0]$. In other words, at $d=d^{*}$ a structural transition takes place in this system of localized charges.

A closer look at the interaction potential $V_{q}^{(f)}$ helps us to estimate the influence of the experimental parameters (holding field $E_{\perp}$ and surface-roughness wave vector $q$ ) on this transition. First we should emphasize that the polarization part $P_{q}^{(f)}(z, d)$ increases with $q^{2}$ and becomes much larger than the holding-field-dependent term in Eq. (5) for typical SE concentrations and large enough $q$, except for the close vicinity of the structural transition at $d=d^{*} \simeq d_{0} \sim q^{-1 / 2}$. Then the position of the retrapping transition at $d^{*}$ is almost independent of $q-\mathrm{a}$ 
a)

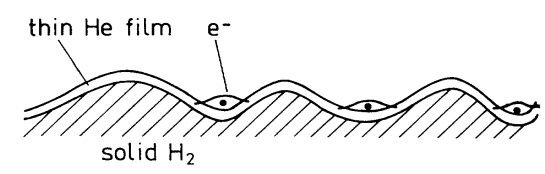

b)

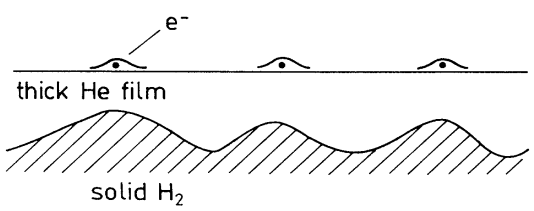

FIG. 1. Schematic sketch of the two states involved in the structural transition of the SE above a rough solid substrate covered with a helium film. (a) SE are localized in the valleys of the surface roughness. (b) SE are localized opposite the tops of the solid surface roughness.

change in $q$ mainly affects the magnitude of the interaction potential. For large enough $q$ this statement will be proved in Sec. III A by a numerical calculation of $V_{q}^{(f)}$. As sketched below, the tendency can be seen even analytically in the limiting case $[q(z+d)]^{2} / 2 \ll 1$ which might just apply to the experimental conditions. We have seen above that in this limit $P_{q}^{(f)}$ is changing sign at already a small disbalance $1-B_{q}$ when all of the terms inside the square brackets of the expression for $P_{q}^{(f)}(z, d)$ in Eq. (5) are approximately independent of $q: Q$ depends only logarithmically on $q, B_{q}$ is close to $1,\left(B_{q}-1\right) / q^{2}$ is approximately constant. Under these circumstances we can greatly simplify our problem by using only one typical absolute value of the wave vector $q$ describing the surface roughness of the solid substrate. A distribution of wave numbers around $q$ will only slightly broaden the transition.

For a quantitative test of this behavior we can use the results of experimental studies on bare hydrogen surfaces, ${ }^{9}$ which indicate a thermally activated behavior of the conductivity $\sigma=\sigma_{0} \exp \left(-\Delta E_{s} / k_{B} T\right)$. The activation energy $\Delta E_{s}$ could be lowered by thermal annealing. For rougher surfaces and small electron densities $n_{s}$ (some $10^{8} \mathrm{~cm}^{-2}$ ) the measured $\Delta E_{s}$ can be considered to be mostly due to the substrate roughness. In the following we consider the simplified model with only a single $q$ dominating the electron-roughness interaction. In order to establish a connection between the activation energy and the surface profile, we note that in the strong-coupling limit the activation energy above a helium film $\Delta E_{f}$ is proportional to the amplitude of the electron-roughness interaction potential $\left\langle 1\left|V_{q}^{(f)}\right| 1\right\rangle$ (we do not have to worry about the long-range order of the potential, because quantum tunneling along the surface is negligible). Since we have to recover the activation energy above the bare hydrogen surface $\Delta E_{s}$ for $d=0$, we can write down the equation with the proportionality constant:

$\Delta E_{f}=\Delta E_{s}\left|v_{q}(d)\right|, \quad v_{q}(d)=\frac{\left\langle 1\left|V_{q}^{(f)}(z, d)\right| 1\right\rangle}{\left\langle 1\left|V_{q}^{(s)}(z)\right| 1\right\rangle}$.

Evidently $v_{q}(d)$ is independent of $\xi_{q}^{(s)}$. It characterizes the change in interaction potential between the SE and the substrate as a function of helium-film thickness. Hence all substrate properties are included in $\Delta E_{s}$ which has been measured experimentally.

We include scattering of the SE from gas atoms by adding an appropriate resistance term $1 / \sigma_{g}$ :

$$
\sigma=\sigma_{0}\left[\exp \left(\left|v_{q}(d)\right| \frac{\Delta E_{s}}{k_{B} T}\right)+\frac{\sigma_{0}}{\sigma_{g}}\right]^{-1},
$$

accounting for the fact that the conductivity is limited chiefly by gas atom scattering in the region where the surface-roughness contribution is small. One should note that Eq. (7) must not be considered too seriously in the close vicinity of the conductivity maximum, since $\Delta E_{s}\left|v_{q}(d)\right|<k_{B} T$ for small interaction potentials, i.e., the thermal activation picture breaks down and Eq. (7) is not strictly valid anymore. However, at any other film thickness $d$ where $\Delta E_{s}\left|v_{q}(d)\right|>k_{B} T$, including the conductivity dip, expression (7) represents a good approximation.

\section{RESULTS AND DISCUSSION}

\section{A. The interaction potential}

Within the interesting range of film thickness $d$ there are no a priori small parameters in our problem: the average distance $\bar{z}$ of the SE from the liquid-helium surface is usually on the same order of magnitude as $d$; furthermore the roughness wave number $q$ cannot be assumed small compared to $1 /(z+d)$ for the whole range of film thickness. Thus the averaging of the interaction potential $V_{q}^{(f)}(z, d)$ over the SE ground-state wave function $\langle 1 \mid z\rangle=2 a^{-3 / 2} z \exp (-z / a)$ and the calculation of the film-thickness-dependent effective Bohr radius $a(d)$ which enters here have to be done numerically. The resulting electron-roughness interaction potential $v_{q}(d)$ is given in Fig. 2 for different roughness wave numbers $q$, normalized to its value on the bare hydrogen surface. Here we use the dimensionless film thickness $d / a_{s}$, where $a_{s}=15.7 \AA$ is the effective Bohr radius above bare hydrogen. The positions of the extrema of $\left|v_{q}(d)\right|$, i.e., the minimum at $d=d^{*}$ and the maximum at $d=d_{m}>d^{*}$, depend on the roughness wave number $q$ (especially for small $q$ ), shifting toward smaller thickness with increasing $q$. The potential $v_{q}(d)$ which weakly depends on the holding field $E_{\perp}$ in the long-wavelength limit $\left(q<\pi \times 10^{5} \mathrm{~cm}^{-1}\right)$ becomes field independent for short-wavelength surface roughness $\left(q>3 \pi \times 10^{5} \mathrm{~cm}^{-1}\right)$ at least for small SE concentrations $n_{s} \leq 3 \times 10^{8} \mathrm{~cm}^{-2}$. As another important consequence from the above results the values of $d^{*}$ and $d_{m}$ must be independent of temperature and the surface-roughness amplitude, which is changed during annealing. 


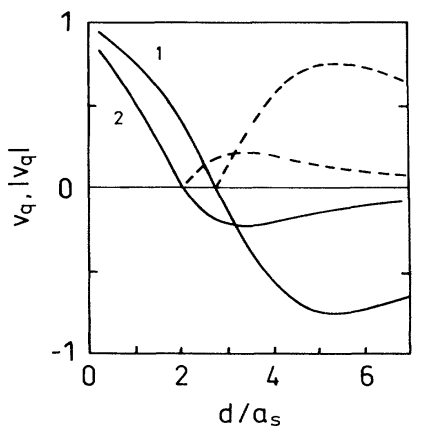

FIG. 2. Electron-roughness interaction potential $v_{q}(d)$ (solid) from Eq. (6) and its absolute value $\left|v_{q}(d)\right|$ (dashed) as a function of dimensionless film thickness $d / a_{s}$ for a SE concentration of $n_{s}=3.1 \times 10^{8} \mathrm{~cm}^{-2}$ and roughness wave numbers $q=\pi \times 10^{5} \mathrm{~cm}^{-1}$ (1) and $q=3 \pi \times 10^{5} \mathrm{~cm}^{-1}$ (2).

In order to determine the most relevant $q$ for SE above quench-condensed hydrogen films, we compare our conductivity calculations with the experimental data. The results of numerical calculations based on Eqs. (5)-(7) are presented in Fig. 3(a) for an activation energy $\Delta E_{s}=18 \mathrm{~K}$ and an electron density of $n_{s} \leq 3.1 \times 10^{8} \mathrm{~cm}^{-2}$, corre-
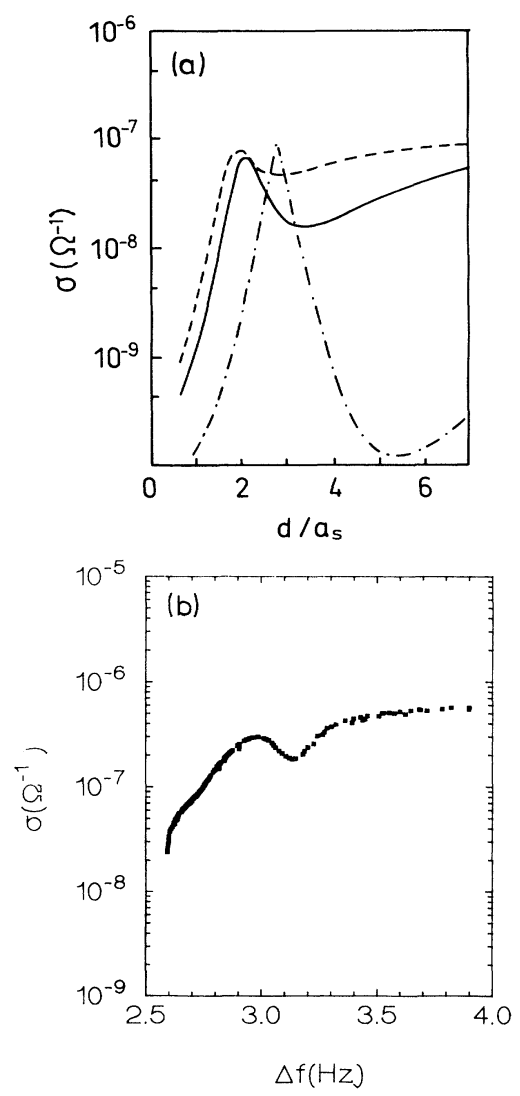

FIG. 3. (a) SE conductivity $\sigma$ vs $d / a_{s}$ (taking $\Delta E_{s}=18 \mathrm{~K}$ ) for three values of $q: \pi \times 10^{5} \mathrm{~cm}^{-1}$ (dashed-dotted), $3 \pi \times$ $10^{5} \mathrm{~cm}^{-1}$ (solid), and $5 \pi \times 10^{5} \mathrm{~cm}^{-1}$ (dashed). (b) Experimental SE conductivity data vs frequency of a quartz balance measuring the helium-film thickness. sponding to the experimental situation in Ref. 6. In an actual experiment $\Delta E_{s}$ will be influenced by the electric holding field as well as by correlation within the electron system, which will be discussed below. Comparing our calculations with experimental conductivity data (shown in Fig. 3) from a system with conditions similar to the ones used in the above calculation, we can estimate the dominant surface-roughness wave number to be typically around or above $3 \pi \times 10^{5} \mathrm{~cm}^{-1}$. For such rather short wavelengths the position of the conductivity maximum $d^{*}$ is quite independent of $E_{\perp}$ in accordance with the experiment. As shown in Sec. II, this diminishing influence of $E_{\perp}$ is a consequence of the strong increase of the polarization term in Eq. (5) in the short-wavelength limit $\left(P_{q}^{(f)} \sim q^{2}\right.$ for $\left.q z \ll 1\right)$. The maximum position also shows only a rather weak dependence on $q$ in this wavelength region, again justifying the harmonic description we are using in our model for the solid roughness.

\section{B. Comparison with temperature-dependent data}

Unfortunately most of the relevant experimental data are recorded as isochores, i.e., the helium-film thickness is changed by varying the temperature $T$ in a closed experimental cell. In order to determine the film thickness $d(T)$, in general one must take into account that the amount of helium gas above the film self-consistently depends on $d$. Luckily, for $q=3 \pi \times 10^{5} \mathrm{~cm}^{-1}$ the positions $d^{*}=35 \AA$ for the conductivity maximum and $d_{m}=56 \AA$ for the dip are far enough away from the saturated film thickness $\left(d_{\text {sat }} \simeq 270 \AA\right)$, i.e., it is sufficiently accurate to use the quite simple van der Waals relation

$$
d^{-3}(T)=\frac{k_{B} T}{A_{H}}\left[\frac{3}{2} \ln \left(\frac{T}{T_{c}}\right)+W \frac{T-T_{c}}{k_{B} T_{c} T}\right]
$$

based on the usual temperature dependence of the saturated helium gas concentration $n_{G}^{\text {(sat) }}(T) \sim$ $T^{3 / 2} \exp \left(-W / k_{B} T\right)$, where $W / k_{B}=7.17 \mathrm{~K}$ is the evaporation energy of a helium atom from the surface. In this approximation we get $d \rightarrow \infty$ for $T \rightarrow T_{c}$, taking the gas atom concentration $n_{G}$ as fixed. The critical temperature $T_{c}$ is determined by the total amount of helium in the cell.

The resulting conductivity $\sigma(T)$ is presented in Fig. 4(a) for $T_{c}=1.97 \mathrm{~K}$ and different annealing conditions represented by the activation energy $\Delta E_{s}$. Figure 4(b) shows the corresponding experimental data from Ref. 6 for comparison. As can be seen, theory and experiment are remarkably close, both showing a suppression of the dip on progressive annealing while the dip position remains unaffected. The theory gives a slightly sharper structure of the anomaly, since we used a fixed value for $q$, whereas in reality a range of $q$ values contributes to the signal. The most notable difference between the calculated and measured data appears near the conductivity maximum (especially for the smallest value of $\Delta E_{s}$ ) where Eq. (7) fails, and at $T \simeq T_{c}$ where Eq. (8) is not valid anymore. Nevertheless the theory agrees surprisingly well with the experiment, even quantitatively, 

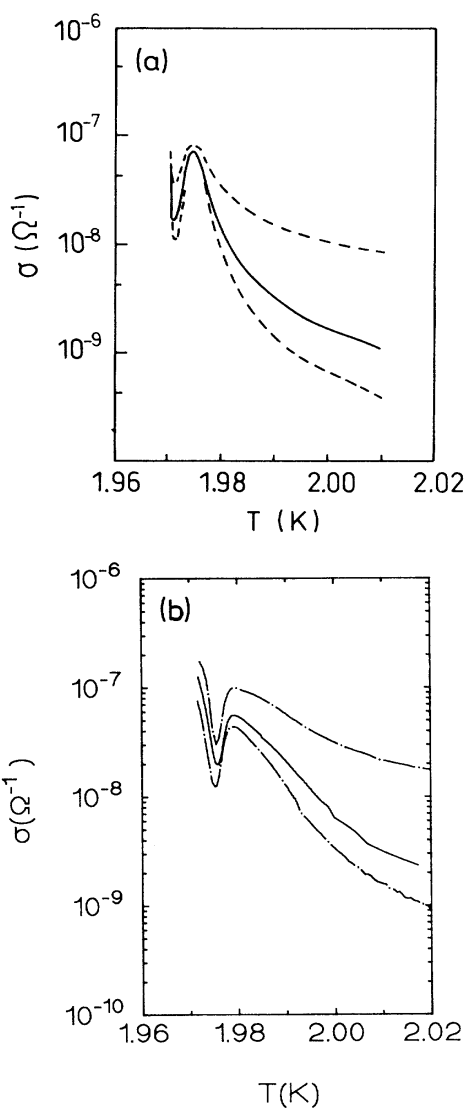

FIG. 4. SE conductivity as a function of temperature in an isochoric environment for activation energies $\Delta E_{s}=22 \mathrm{~K}$ (upper dashed line), $18 \mathrm{~K}$ (solid line), and $10 \mathrm{~K}$ (lower dashed line). (a) Results of the calculations, (b) experimental data from Ref. 6.

reproducing not only the dip structure but also the hightemperature tail of the conductivity as a function of different annealing degrees.

\section{Changing the surface-roughness wave vector}

It should be noted here that from the physical point of view the conductivity maximum is a more important and unexpected feature than the dip, because it corresponds to a specific structural transition in the electron system from a strong $\mathrm{SE}$ localization in the valleys to a strong localization above the tops of the solid surface-roughness profile. At the transition itself, i.e., at the conductivity maximum, the SE become nearly free. Figure 5 shows $\sigma(T)$ for a freshly prepared quench-condensed hydrogen film without any annealing which is extremely rough. Indeed the maximum appears as a very pronounced feature, the conductivity at the maximum attaining nearly the same value as on the saturated helium film. The very sharp tip of the maximum indicates that the electrons mostly occupy rather long-wavelength potential wells of the surface [cf. Fig. 3(a)]. Data taken after annealing,

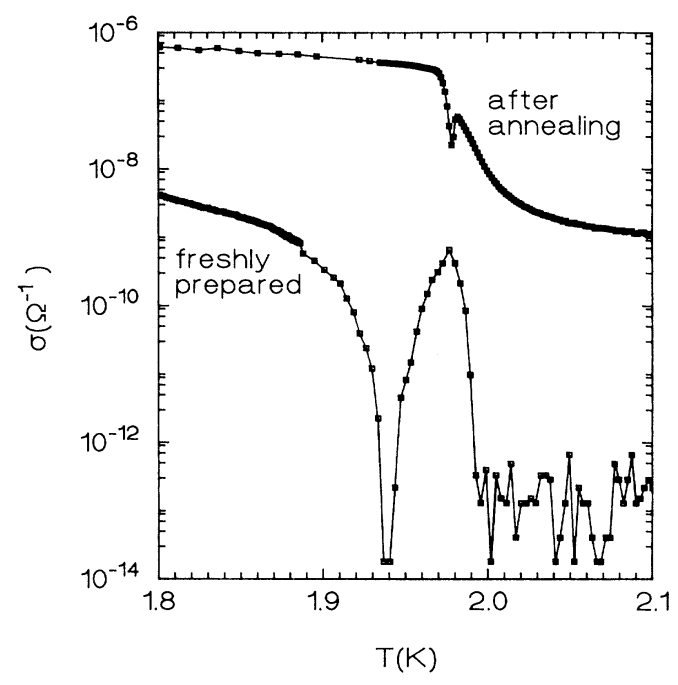

FIG. 5. Conductivity anomaly on a freshly prepared quench-condensed hydrogen film (lower curve) and after thermal annealing (upper curve).

also shown in Fig. 5, exhibit a much smoother maximum which is shifted to smaller thickness values (i.e., higher $T$ ); the conductivity minimum is shifted even more strongly towards higher $T$. We conclude that in our case the annealing process reduces the long-wavelength roughness - the observed behavior is then in perfect accordance with the theoretical results presented in Figs. 2 and 3.

\section{Influence of correlation effects}

Correlation effects within the electron system become increasingly important with higher electron density $n_{s}$. Even for the rather low electron densities we have dealt with above the plasma parameter $\Gamma=\sqrt{\pi n_{s}} e^{2} / \epsilon k_{B} T$ (where $\epsilon$ is the effective dielectric constant of the substrate) is larger than 10 , and it attains values above 100 for $n_{s} \simeq 10^{10} \mathrm{~cm}^{-2}$. Hence correlation effects must be taken into account. For delocalized electrons there is no significant dependence of the SE mobility on $\Gamma .{ }^{10}$ The situation is changed, however, if the electrons are in localized states as in our case. Due to their trapping at surface heterogeneities the electrons might evolve into a sort of glassy state due to their mutual interaction. In this picture electron hopping is suppressed by the additional Coulomb energy arising as a consequence of the correlation. We can estimate this correlation correction $\Delta E_{c}$ to the activation energy $\Delta E_{s}$ in the limiting case $\lambda=2 \pi / q \ll \bar{a}=1 / \sqrt{n_{s}}$ as

$$
\Delta E_{c} \simeq \frac{\pi}{2} e^{2} \frac{n_{s}}{\bar{a}} \lambda^{2} .
$$

For densities of $n_{s}=10^{9} \mathrm{~cm}^{-2}$ and $q=3 \pi \times 10^{5} \mathrm{~cm}^{-2}$ it attains a value of about $7 \mathrm{~K}$. At even higher electron densities where $\lambda \simeq \bar{a}$ correlations become more effective -in this limiting case $\Delta E_{c} \sim e^{2} / \bar{a} \sim \Gamma k_{B} T \gg k_{B} T$. A simi- 


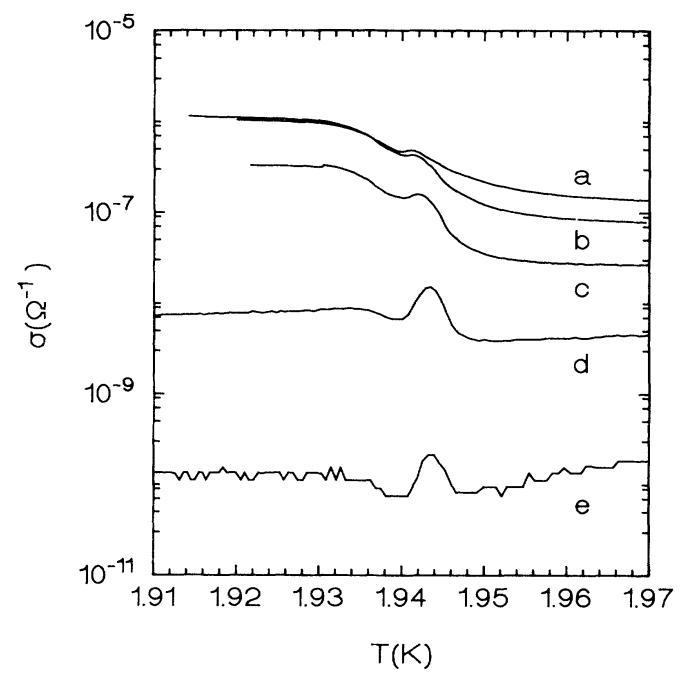

FIG. 6. Conductivity anomaly on an annealed hydrogen film $\left(\Delta E_{s} \simeq 5 \mathrm{~K}\right)$ for various electron densities $n_{s}$ given below; the corresponding approximate values for the plasma parameter $\Gamma$ are shown in parentheses. $a, 5.1 \times 10^{8} \mathrm{~cm}^{-2}$ $(\Gamma \simeq 30) ; b, 2.0 \times 10^{9} \mathrm{~cm}^{-2}(\Gamma \simeq 55) ; c, 4.6 \times 10^{9} \mathrm{~cm}^{-2}$ $(\Gamma \simeq 85) ; d, 8.1 \times 10^{9} \mathrm{~cm}^{-2}(\Gamma \simeq 115) ; e, 1.27 \times 10^{10} \mathrm{~cm}^{-2}$ $(\Gamma \simeq 140)$.

lar dependence of correlation on the SE mobility was in fact observed for SE on a bare hydrogen film. ${ }^{11}$ Consequently, the SE mobility at both sides of the conductivity maximum should be strongly suppressed for high electron densities. New studies of the SE conductivity near the maximum for various electron densities (Fig. 6) demonstrate such a behavior. The data were collected in the same way as described in Ref. 6 . The corresponding data on a bare hydrogen film ${ }^{11}$ as expected show a thermally activated behavior where the activation energy increases with correlation.

\section{CONCLUSIONS}

We have investigated the conductivity behavior of surface electrons on an uneven solid hydrogen substrate covered with a thin helium-film in the limit of strong coupling. Upon increasing the helium-film thickness the electron system undergoes a structural transition from a state where the electrons are trapped in the valleys of the surface roughness profile to a state where they are localized on a thick helium film opposite the tops of the profile. At the transition itself the SE become nearly free. From the electron-roughness interaction potential we can calculate the SE conductivity as a function of helium-film thickness. Using parameters measured in previous experiments we can quantitatively explain almost all features of the conductivity anomaly observed on rough hydrogen substrates (the concept should apply equally to any other substrate where the anomaly has been observed). Experimental data measuring the dependence of the transition on changes in the surface-roughness wave vector and on correlation effects within the electron system lend additional support to the validity of the presented calculations.

\section{ACKNOWLEDGMENTS}

U.A., K.K., and P.L. acknowledge financial support from the SFB306 of the Deutsche Forschungsgemeinschaft. K.K. acknowledges support from the Alexander von Humboldt-Stiftung.
*Present address: Institute for Solid State Physics, University of Tokyo, Roppongi, Tokyo 106, Japan

${ }^{\dagger}$ Author to whom all correspondence should be addressed.

${ }^{1}$ E.Y. Andrei, Phys. Rev. Lett. 52, 1449 (1984).

${ }^{2}$ K. Kajita and W. Sasaki, Surf. Sci 113, 419 (1982).

${ }^{3}$ M.A. Paalanen and Y. Iye, Phys. Rev. Lett. 55, 1761 (1985).

${ }^{4}$ D. Cieslikowski, A.J. Dahm, and P. Leiderer, Phys. Rev. Lett. 58, 1751 (1987).

${ }^{5}$ U. Albrecht, H. Dilger, K. Kono, and P. Leiderer, Physica B 165\&166, 841 (1990).
${ }^{6} \mathrm{~K}$. Kono, U. Albrecht, and P. Leiderer, J. Low Temp. Phys. 83, 423 (1991).

${ }^{7}$ V.B. Shikin and P. Leiderer, Pis'ma Zh. Eksp. Teor. Fiz. 53, 449 (1991) [JETP Lett. 53, 426 (1991)].

${ }^{8}$ Yu.P. Monarkha, Fiz. Nizk. Temp. 18, 210 (1992).

${ }^{9} \mathrm{~K}$. Kono, U. Albrecht, and P. Leiderer, J. Low-Temp. Phys. 82, 279 (1991).

${ }^{10}$ R. Mehrotra, C.J. Guo, Y.Z. Ruan, D.B. Mast, and A.J. Dahm, Phys. Rev. B 29, 5239 (1984).

${ }^{11}$ F. Mugele, U. Albrecht, P. Leiderer, and K. Kono, J. LowTemp. Phys. 89, 743 (1992). 